\title{
Friction of two-dimensional colloidal particles with magnetic dipole and Lennard-Jones interactions: A numerical study
}

\author{
Zhongying ZHANG, Cange WU, Qi ZHANG, Yigang CAO* \\ School of Physics and Engineering, Zhengzhou University, Zhengzhou 450001, China \\ Received: 13 November 2018 / Revised: 15 January 2019/Accepted: 16 February 2019 \\ (C) The author(s) 2019.
}

\begin{abstract}
We use Langevin simulations to study the sliding friction of two-dimensional colloidal particles on a substrate with randomly distributed point-like pinning centers. The colloidal particles are modeled to interact with each other through repulsive magnetic dipole and attractive Lennard-Jones potentials. The subsequent occurrence of superlubricity, wherein the average friction force equals to zero, is accompanied by the appearance of islands with clear boundaries in the microscopic colloidal structures for weak pinning substrates. Friction arises for strong pinning substrates, and the average friction force increases with the substrate pinning intensity, and further, the islands disperse into disordered plastic structures. Moreover, the average friction force decreases with the repulsion intensity between the colloidal particles, and superlubricity finally results when the repulsion becomes sufficiently strong. Superlubricity also occurs for sufficiently weak attraction between colloidal particles, with an increase in the attraction intensity between colloidal particles leading to a nonlinear increase in the average friction force. With increasing temperature, the average friction force firstly increases and subsequently decreases rapidly. The above results can provide a theoretical framework for biological self-organization via utilization of the friction properties of microscopic or mesoscopic colloidal systems.
\end{abstract}

Keywords: friction; sliding friction; superlubricity; self-assembly

\section{Introduction}

Friction plays a central role in various systems that range from the nanometer to geophysical scales [1]. Friction is encountered in all aspects of science and technology, and this has led to continual advancements in friction studies over centuries. In this context, developments in nanotechnology permit the study of friction at the nano/micro scales, and studies of nanofriction can provide greater insights into the microscopic nature of friction [1-3].

Meanwhile, colloidal suspensions provide unique possibilities for obtaining mesoscopic information about nature, for, e.g., mesoscopic friction behaviors, because the size of colloidal particles lies in the range between nanometers and micrometers. Some colloidal particles are superparamagnetic, and the magnitude of the induced magnetic moment in these colloidal particles scales linearly with the applied magnetic field strength [4-8]. Thus, the interaction between magnetized colloidal particles has the form of a repulsive magnetic dipole potential. Here, we note that magnetic gels can be easily prepared [9] for use in experiments, and the dynamic properties of magnetized colloids are more abundant than those of charged colloids [10-13], for, e.g., there are richer dynamic phase transitions near depinning in the magnetized colloids [13]. In this study, we focus on magnetized colloids.

Recently, active colloids have attracted considerable research interest. Active systems can actively absorb energy from the environment and overcome resistance (i.e., energy barrier) through energy storage [14], as in the case of bacterial colonies of fish and birds and tissues of cells; such systems are different from passive

* Corresponding author: Yigang CAO, E-mail: physycao@zzu.edu.cn 
systems that acquire energy from the surrounding environment and subsequently restore energy to the environment. It is known that the abovementioned activity originates from weak attractive interactions between individuals in the systems [15-20], for, e.g., van der Waals interaction between colloidal particles. Such self-propelling interactions between individual members of active systems [21] drive the systems to non-equilibrium states, which leads the systems exhibiting a series of novel behaviors that cannot be observed under equilibrium [20-25]. The most striking behaviors include the formations of clusters with finite size or islands with clear boundaries (the so-called living islands) [20-25]. These structures are closely related to biological self-organization [17, 20-25]; in this context, we note that biological activity is also one of the remarkable characteristics of biotribological problems in relation to general tribological problems.

Currently, nanotribology studies are seeking to explore novel routes, for, e.g., phase transitions [26], to achieve frictional control, which can in turn be used to control biological activity and self-organization [1]. Against this backdrop, here, we numerically investigate the sliding friction of two-dimensional (2D) active magnetized colloids on a substrate with randomly distributed point-like pinning centers. The substrate is a realistic quenched one, which is different from an ordered substrate [27]. The active magnetized colloidal particles are modeled as interacting with each other via repulsive magnetic dipole and attractive LennardJones potentials [20,28]. We attempt to determine the relationship between sliding friction and dynamic phases and phase transitions, which can provide a theoretical framework for the control of biological self-organization via utilization of the friction properties of mesoscopic colloidal systems.

\section{Model}

The system of interest consists of 400 active magnetized colloidal particles, which are modeled to interact with each other through repulsive magnetic dipole and attractive Lennard-Jones potentials. These particles are initially placed in an ideal triangular lattice satisfying periodic boundary conditions. The substrate is simulated by 400 randomly distributed point-like pinning centers. We describe the motion of colloidal particles by means of the Langevin equation [11-13, 17, 20, 28, 29]:

$$
\eta \frac{\mathrm{d} \boldsymbol{R}_{i}}{\mathrm{~d} t}=-\sum_{i \neq j} \nabla_{i} U_{\mathrm{cc}}\left(\boldsymbol{R}_{i}-\boldsymbol{R}_{j}\right)-\sum_{j^{\prime}} \nabla_{i} \mathrm{U}_{\mathrm{cp}}\left(\boldsymbol{R}_{i}-\boldsymbol{r}_{j^{\prime}}\right)+\boldsymbol{F}_{i}^{\mathrm{T}}
$$

where $\eta$ is the viscosity coefficient, and $\boldsymbol{R}_{i}$ and $\boldsymbol{r}_{j^{\prime}}$ are the positions of the $i$-th colloidal particle and $j^{\prime}$-th pinning center, respectively. Further, $U_{\mathrm{cc}}(r)$ with $r=\left|\boldsymbol{R}_{i}-\boldsymbol{R}_{j}\right|$ is the interaction between colloidal particles, including the repulsive component $U_{\mathrm{cc}}^{\text {rep }}(r)$ and attractive component $U_{\mathrm{cc}}^{\text {att }}(r)$. Further, $U_{\mathrm{cp}}\left(r^{\prime}\right)$ with $r^{\prime}=\left|\boldsymbol{R}_{i}-\boldsymbol{r}_{j^{\prime}}\right|$ is the interaction between the colloidal particles and pinning centers in the substrate. $F_{i}^{\mathrm{T}}$ is the thermal force.

When a magnetic field $\boldsymbol{B}$ acts perpendicular to the plane of the system, the induced magnetic dipole potential has the repulsive form [10, 11-13, 20, 28, 29]:

$$
U_{\mathrm{cc}}^{\mathrm{rep}}(r)=\frac{A_{\mathrm{v}}^{2}}{r^{3}}
$$

where $A_{v}=\sqrt{\frac{\mu_{0}}{4 \pi}} \chi B$, with $\mu_{0}$ being the vacuum permeability, $\chi$ being the magnetic susceptibility, and $B$ being the magnetic field strength. Further, $A_{v}$ is the repulsion intensity between colloidal particles, and it is controlled completely by the magnetic field strength $B$.

The attractive interaction $U_{\mathrm{cc}}^{\text {att }}(r)$ is chosen as the Lennard-Jones potential [16, 20, 24, 28]:

$$
U_{\mathrm{cc}}^{\mathrm{att}}(r)=4 A_{\mathrm{e}}\left[\left(\frac{\sigma}{r}\right)^{12}-\left(\frac{\sigma}{r}\right)^{6}\right]
$$

Here, $A_{\mathrm{e}}$ and $\sigma$ are the attraction intensity and diameter of the colloidal particles, respectively.

Next, we choose the interaction between colloidal particles and pinning centers in the substrate, $U_{\mathrm{cp}}\left(r^{\prime}\right)$, as the traditional Gaussian attractive potential [11-13, 20, 28, 29]:

$$
U_{\mathrm{cp}}\left(r^{\prime}\right)=-A_{\mathrm{p}} \mathrm{e}^{-\left(\frac{r^{\prime}}{r_{\mathrm{p}}}\right)^{2}}
$$

where $A_{\mathrm{p}}$ is the substrate pinning intensity, $r_{\mathrm{p}}$ is the radius of the pinning center, and we assume $r_{\mathrm{p}}=0.2 a_{0}$, 
with $a_{0}$ being the lattice constant of the ideal triangular lattice.

The thermal force $\boldsymbol{F}_{i}^{\mathrm{T}}$ describes the coupling process with a heat bath and satisfies [11-13, 20, 28, 29]:

$$
<\boldsymbol{F}_{i}^{\mathrm{T}}(t)>=0
$$

and

$$
<\boldsymbol{F}_{i \alpha}^{\mathrm{T}}(t) \boldsymbol{F}_{j \beta}^{\mathrm{T}}\left(t^{\prime}\right)>=2 \eta T \delta_{i j} \delta_{\alpha \beta} \delta\left(t-t^{\prime}\right)
$$

where subscripts $\alpha$ and $\beta$ are the components of $\boldsymbol{F}_{i}^{\mathrm{T}}$ and $\boldsymbol{F}_{j}^{\mathrm{T}}$, respectively. Further, $T$ is the temperature of system.

We present all quantities in dimensionless units in our simulations. The length is based on the lattice constant $a_{0}$, and we scale the temperature with respect to the bare Kosterlitz-Thouless melting temperature of a pure system, $T_{\mathrm{m} 0}$ [30]. The time scale is chosen as $T_{\mathrm{m} 0} / \eta a_{0}^{2}$. We measure the average friction force as $[1,27]$ :

$$
F_{\mathrm{L}}=-\eta v
$$

with $v$ being the average colloidal velocity along the $x$ symmetric axis. Further, $\Delta t=0.001$ is set as the time integration step, and the averages are evaluated over $2 \times 10^{5}$ steps after setting $1 \times 10^{5}$ steps for equilibrium.

\section{Results and discussion}

\subsection{Zero-temperature case}

Firstly, we investigate the influence of the substrate pinning intensity on the average friction force. Given a low repulsion intensity $\left(A_{\mathrm{v}}=0.1\right)$ and a high attraction intensity $\left(A_{\mathrm{e}}=1000\right)$, we present the pinning intensity $\left(A_{\mathrm{p}}\right)$ dependence of the average friction force $F_{\mathrm{L}}$ in Fig. 1, from which we can clearly infer the superlubricity phenomenon, i.e., $F_{\mathrm{L}} \approx 0$, over a large range of values of $A_{\mathrm{p}}\left(A_{\mathrm{p}}<4\right)$. Islands with clear boundaries (i.e., living islands [16]) and separation between different islands are observed in the microscopic structures of colloidal particles for sufficiently weak pinning substrates $\left(A_{\mathrm{p}} \leq 0.1\right)$, as shown in Fig. 2(a), where the attraction between colloidal particles is dominant and all the colloidal particles are organized

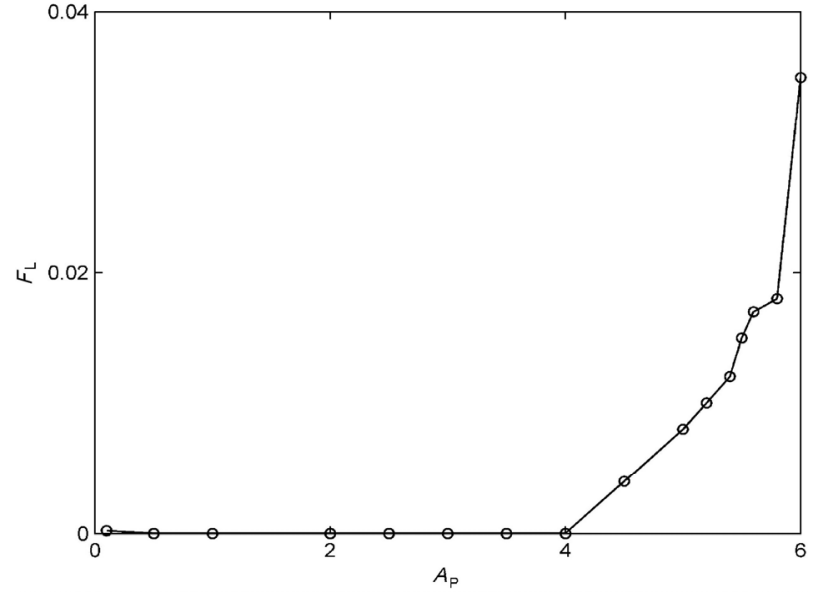

Fig. 1 Dependence of average friction force on substrate pinning intensity.
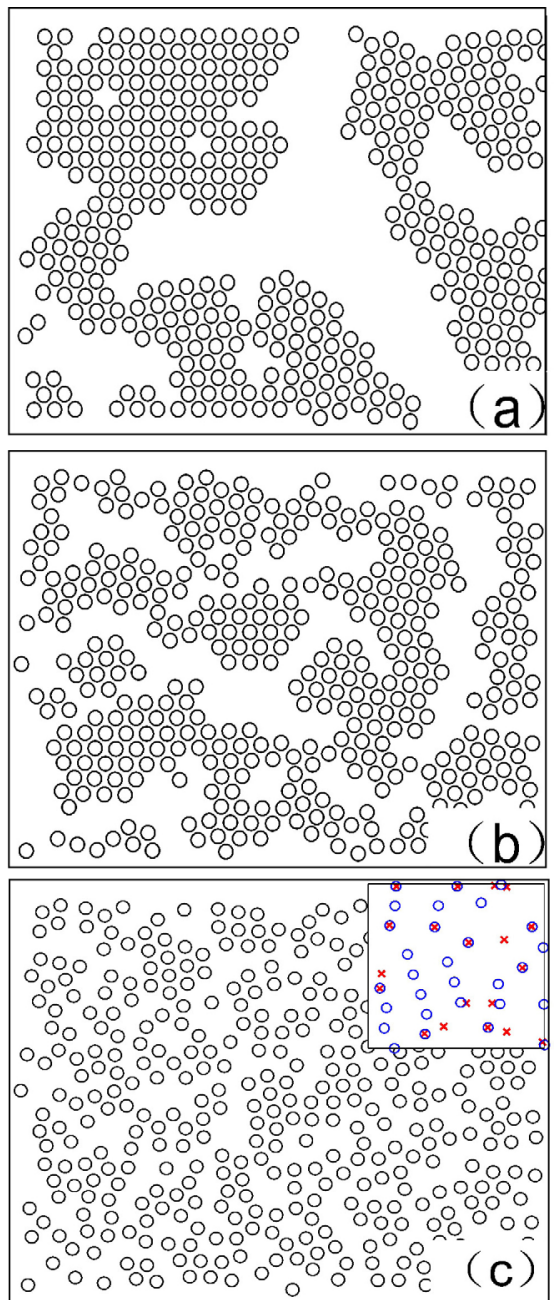

Fig. 2 Coordinates of colloidal particles for (a) $A_{\mathrm{p}}=0.1$, (b) 0.5 , and (c) 5. The inset of (c) shows a snapshot of (c), with the substrate pinning centers denoted by crosses, and free colloidal particles are clearly visible in the channels within different pinning centers. 
into living islands. With increase in the pinning intensity $\left(0.1<A_{\mathrm{p}} \leq 4\right)$, the attraction between colloidal particles and pinning centers in the substrate becomes comparable with the attraction between colloidal particles, and the living islands are dispersed, as shown in Fig. 2(b). In such a case, there is no free particle in the channels within different pinning centers and the superlubricity is still maintained.

As $A_{\mathrm{p}}$ is further increased $\left(A_{\mathrm{p}}>4\right)$, the attraction between colloidal particles and pinning centers in the substrate overcomes the attraction between colloidal particles, and the island structures are destroyed, as shown in Fig. 2(c). Some colloidal particles escape from the islands and lie in the channels within different pinning centers, thus leading to the occurrence of friction. Parameter $F_{\mathrm{L}}$ firstly exhibits a basic linear increase and subsequently increases rapidly when $A_{\mathrm{p}}$ is increased to $\sim 6$, as shown in Fig. 1 .

Next, we examine the influence of the repulsion intensity between colloidal particles on the sliding friction. For a large pinning intensity $\left(A_{\mathrm{p}}=5\right)$ and a large attraction intensity between colloidal particles $\left(A_{\mathrm{e}}=1000\right)$, the repulsion intensity $\left(A_{\mathrm{v}}\right)$ dependence of the average friction force $F_{\mathrm{L}}$ is presented in Fig. 3 . We observe friction for sufficiently small values of $A_{\mathrm{v}}$, and the colloidal particles are in the destroyed island structures, as shown in Fig. 4(a). In such a case, the repulsion between colloidal particles is so small that it can be neglected, and the attraction between colloidal particles and substrate pinning centers becomes comparable with the attraction between colloidal particles, thereby resulting in some colloidal particles

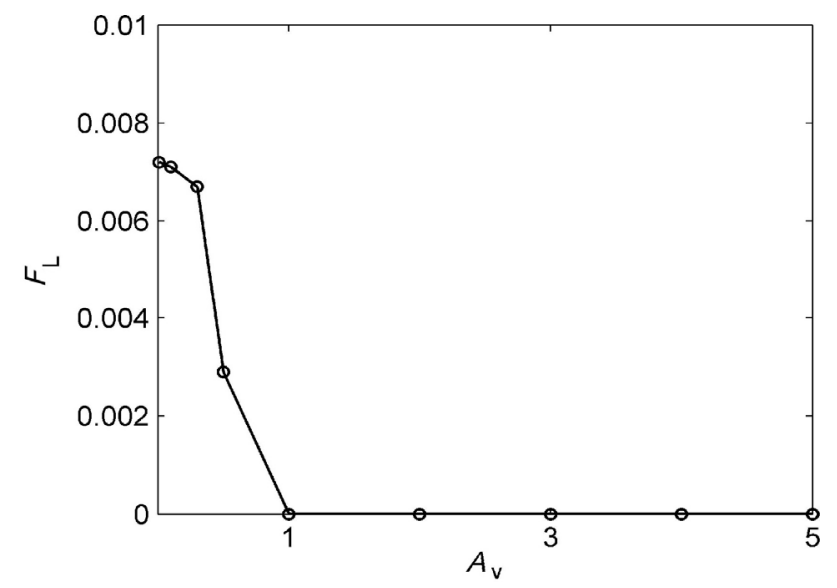

Fig. 3 Dependence of average friction force on repulsion intensity.

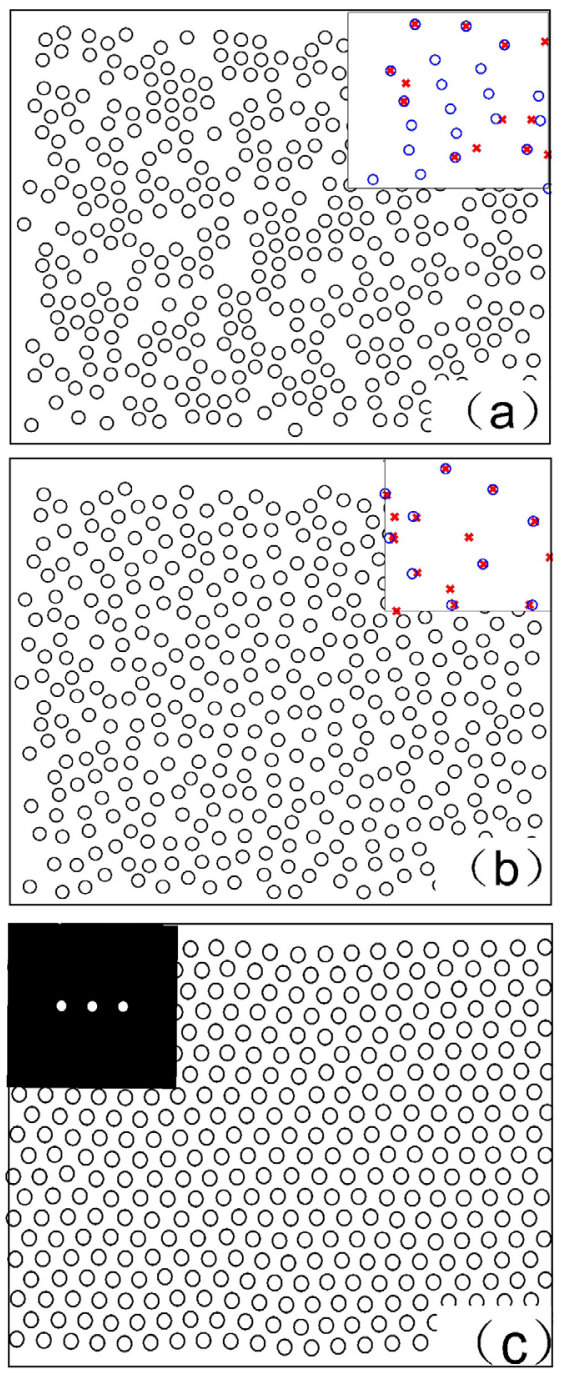

Fig. 4 Coordinates of colloidal particles for (a) $A_{\mathrm{v}}=0.01$, (b) 1 , and (c) 5 . The inset of (c) shows its corresponding structure factor defined as $S(\boldsymbol{k})=\left\langle\left|\frac{1}{N_{\mathrm{c}}} \sum_{i=1}^{N_{\mathrm{c}}} \exp \left(i \boldsymbol{k} \cdot \boldsymbol{R}_{i}\right)\right|^{2}\right\rangle$, where $N_{\mathrm{c}}$ is the number of colloidal particles. The insets of (a) and (b) show the snapshots of (a) and (b), respectively, with the substrate pinning centers denoted by crosses.

moving within different pinning centers, as shown in the inset of Fig. 4(a).

With increase in $A_{\mathrm{v}}$, the repulsion between colloidal particles increases, and the colloidal particles in the channels within different pinning centers repulse each other to be close to the pinning centers, causing a decrease in the friction. Superlubricity occurs when there are a few free colloidal particles in the channels within different pinning centers, as shown in the inset of Fig. 4(b), and uniformly plastic structures appear, as shown in Fig. 4(b). A further increase in $A_{v}$ leads 
to $F_{\mathrm{L}} \approx 0$, i.e., superlubricity, and the colloidal particles are in ordered smectic structures, as shown in Fig. 4(c) and its inset, when $A_{\mathrm{v}}$ is increased to be sufficiently large and the repulsion between colloidal particles dominates.

Next, we study the influence of the attraction intensity between colloidal particles on the friction force. Figure 5 presents the attraction intensity $\left(A_{\mathrm{e}}\right)$ dependence of the average fiction force $F_{\mathrm{L}}$ for $A_{\mathrm{p}}=5$ and $A_{\mathrm{v}}=0.1$. Superlubricity occurs over a range of small values of $A_{\mathrm{e}}$, wherein the colloidal particles assume the form of disordered plastic structures, as shown in Fig. 6(a), and there is no free colloidal particle moving in the channels within different pinning centers, as shown in the inset of Fig. 6(a). As $A_{\mathrm{e}}$ is increased above a certain value $(\approx 300)$, friction arises when the attraction between colloidal particles is comparable with the attraction between colloidal particles and pinning centers in the substrate. As a result, some colloidal particles escape from the pinning centers and move along the channels within different pinning centers, as shown in the inset of Fig. 6(b). Further increasing $A_{\mathrm{e}}$ finally saturates the average friction force $F_{\mathrm{L}}$ to a stable value when the number of colloidal particles within pinning centers remains unchanged, and the colloidal particles are still in form of the disordered plastic structures, as can be observed in Fig. 6(c).

\subsection{Finite-temperature case}

Finally, we investigate the temperature effect on the sliding friction. The temperature dependence of the average friction force $F_{\mathrm{L}}$ is shown in Fig. 7 for $A_{\mathrm{p}}=5$

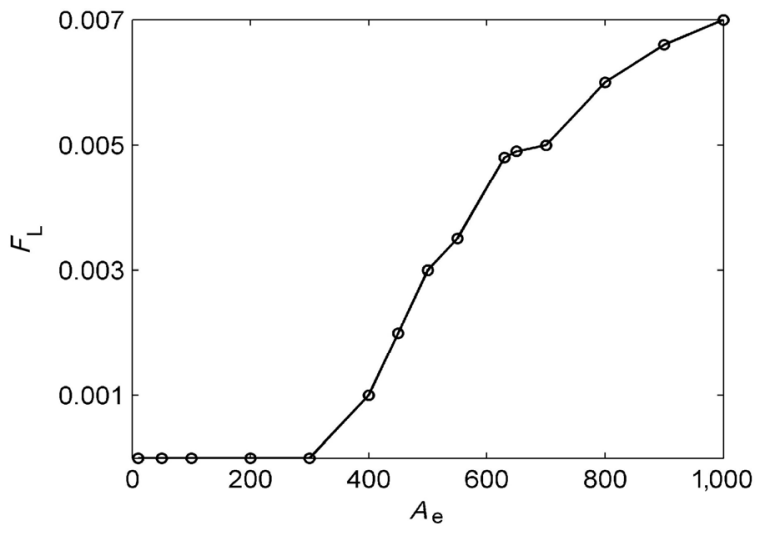

Fig. 5 Dependence of average friction force on attraction intensity.
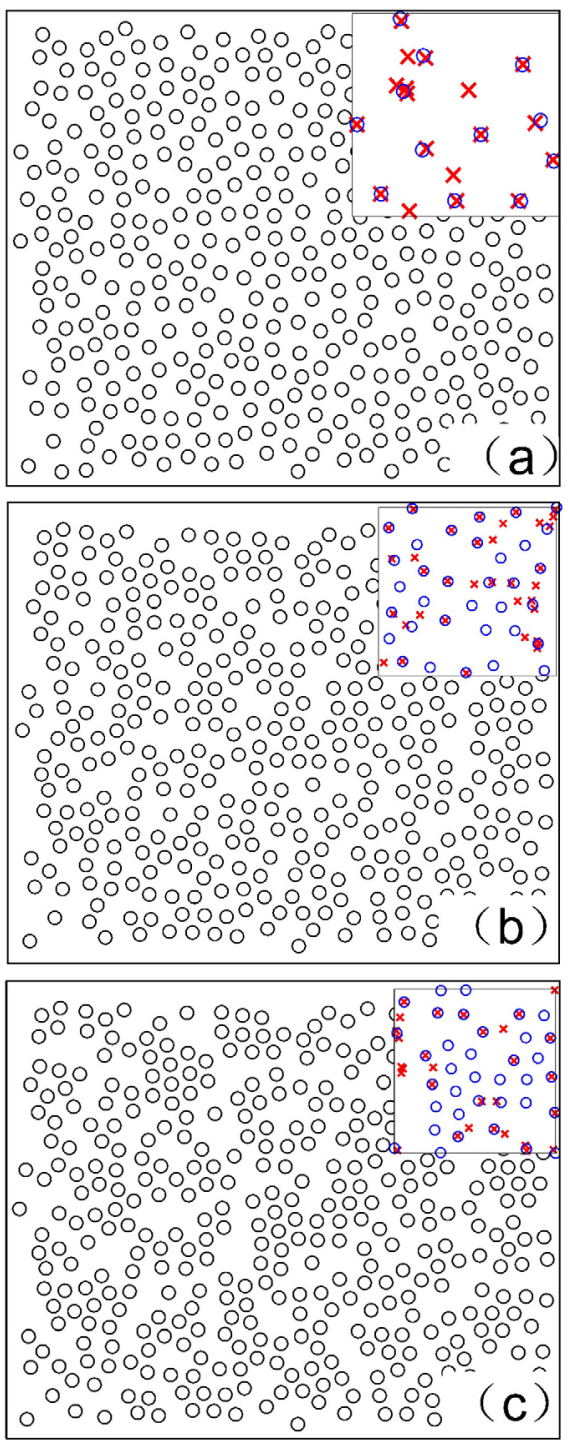

Fig. 6 Coordinates of colloidal particles corresponding to (a) $A_{\mathrm{e}}=10$, (b) 500, and (c) 1000. The insets of (a), (b), and (c) show the snapshots of (a), (b), and (c), respectively, with the substrate pinning centers denoted by crosses.

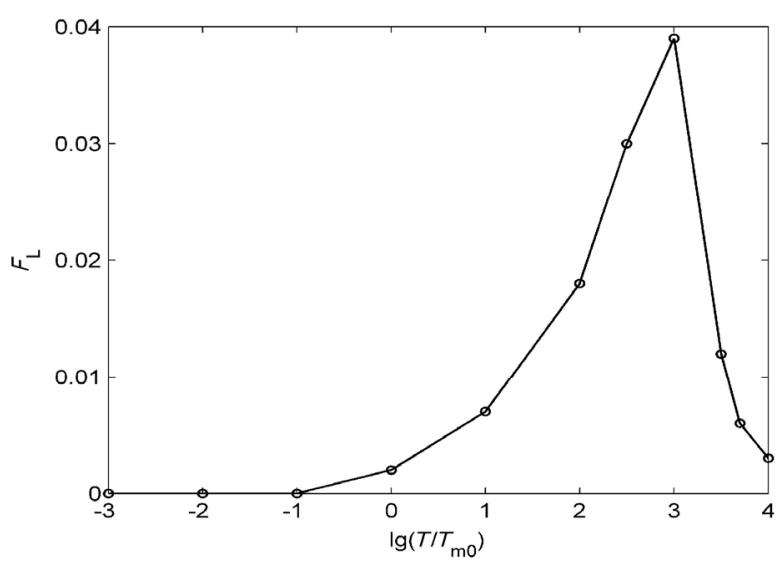

Fig. 7 Dependence of average friction force on temperature. 
and $A_{\mathrm{e}}=1000$. The superlubricity states appear at low temperatures $\left(T<T_{\mathrm{m} 0}\right)$ because the thermal fluctuations are so small that they are negligible in relation to the attraction of pinning centers and there are few free colloidal particles moving in the channels within different pinning centers, as shown in the inset of Fig. 8(a).

With increase in the temperature $\left(T_{\mathrm{m} 0}<T<1000 T_{\mathrm{m} 0}\right)$, the thermal fluctuation increases, and some colloidal particles undergo activation to escape from the pinning
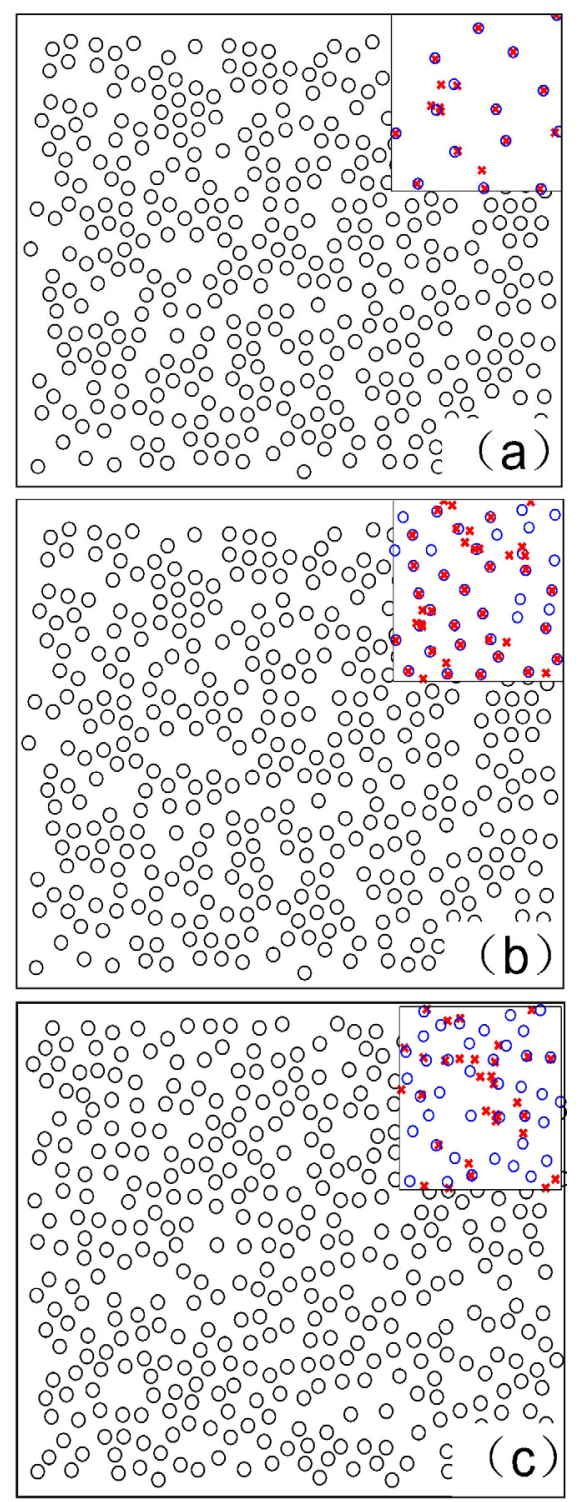

Fig. 8 Coordinates of colloidal particles at different temperatures corresponding to (a) $T=0.001 T_{\mathrm{m} 0}$, (b) $T=T_{\mathrm{m} 0}$, and (c) $T=1000 T_{\mathrm{m} 0}$. The insets of (a), (b), and (c) show the snapshots of (a), (b), and (c), respectively, with the substrate pinning centers denoted by crosses. centers and move into the channels within different pinning centers, as shown in the inset of Figs. 8(b) and $8(\mathrm{c})$, thereby leading to the appearance of friction. The number of colloidal particles within different pinning centers increases with the temperature, thus leading to increase in $F_{\mathrm{L}}$ with temperature. However, with further increase in the temperature $\left(T>1000 T_{\mathrm{m} 0}\right)$, the thermal fluctuations become so large that the pinning potential is smoothed. This leads to a rapid decrease in the average friction force.

\section{Conclusions}

We numerically investigated the sliding friction behavior of 2D colloidal particles with magnetic dipole and Lennard-Jones interactions on a disordered substrate. At low temperatures $\left(T<T_{\mathrm{m} 0}\right)$, the superlubricity phenomenon appears for weak pinning substrates $\left(A_{\mathrm{p}}<4\right)$ and weak attraction between colloidal particles $\left(A_{\mathrm{e}}<300\right)$ as well as for strong repulsion between colloidal particles $\left(A_{\mathrm{v}}>1\right)$. Superlubricity is accompanied by the emergence of islands with clear boundaries in the microscopic colloidal structures for the weak pinning substrates. The observed friction is due to the free colloidal particles moving in the channels within different pinning centers. The average friction force increases nonlinearly with the substrate pinning intensity and the repulsion and attraction intensities between colloidal particles. The thermal fluctuation increases with temperature, thereby leading to an increase in the average friction force below a certain temperature $\left(T \approx 1000 T_{\mathrm{m} 0}\right)$, above which thermal lubrication takes place and the average friction force decreases rapidly.

Our results are valuable in terms of understanding the mechanism of sliding friction in active mesoscopic particle systems and for controlling biological selforganization by exploiting the friction properties of mesoscopic particle systems.

\section{Acknowledgements}

This work was supported by the Postgraduate Education Reform Project of Henan Province under Grant No. 2017SJGLX011Y and Postgraduate Education Research Project of Zhengzhou University under Grant No. 
YJSJY201758 as well as University Students' Innovative Entrepreneurial Project under Grant No. 2017cxcy20.

Open Access: This article is licensed under a Creative Commons Attribution 4.0 International License, which permits use, sharing, adaptation, distribution and reproduction in any medium or format, as long as you give appropriate credit to the original author(s) and the source, provide a link to the Creative Commons licence, and indicate if changes were made.

The images or other third party material in this article are included in the article's Creative Commons licence, unless indicated otherwise in a credit line to the material. If material is not included in the article's Creative Commons licence and your intended use is not permitted by statutory regulation or exceeds the permitted use, you will need to obtain permission directly from the copyright holder.

To view a copy of this licence, visit http://creativecommons.org/licenses/ by/4.0/.

\section{References}

[1] Vanossi A, Manini N, Urbakh M, Zapperi S, Tosatti E. Colloquium: Modeling friction: From nanoscale to mesoscale. Rev Mod Phys 85(2): 529-548 (2013)

[2] Persson B N J. Sliding Friction, Physical Principles and Applications. Berlin: Springer, 2000

[3] Urbakh M, Meyer E. The renaissance of friction. Nat Mater 9(1): 8-10 (2010)

[4] Löwen H. Colloidal soft matter under external control. J Phys: Condens Matter 13(24): R415-R432 (2001)

[5] Löwen H, Messina R, Hoffmann N, Likos C N, Eisenmann C, Keim P, Gasser U, Maret G, Goldberg R, Palberg T. Colloidal layers in magnetic fields and under shear flow. J Phys: Condens Matter 17(45): S3379 (2005)

[6] Köppl M, Henseler P, Erbe A, Nielaba P, Leiderer P. Layer reduction in driven 2D-colloidal systems through microchannels. Phys Rev Lett 97(20): 208302 (2006)

[7] Ebert F, Keim P, Maret G. Local crystalline order in a 2D colloidal glass former. Eur Phys J E 26(1-2): 161-168 (2008)

[8] Ebert F, Maret G, Keim P. Partial clustering prevents global crystallization in a binary 2D colloidal glass former. Eur Phys J E 29(3): 311-318 (2009)

[9] Ebert F, Dillmann P, Maret G, Keim P. The experimental realization of a two-dimensional colloidal model system. Rev Sci Instrum 80(8): 083902 (2009)
[10] Löwen H. Colloidal dispersions in external fields: recent developments. J Phys: Condens Matter 20(40): 404201 (2008)

[11] Cao Y G, Li Q X. Dynamics of magnetized colloids on a disordered substrate. Physica A 387(19-20): 4755-4759 (2008)

[12] Cao Y G, Li Q X, Fu G Y, Liu J, Hu X, Li X J. Depinning dynamics of two-dimensional magnetized colloids on a random substrate. J Phys: Condens Matter 22(15): 155101 (2010)

[13] Cao Y G, Zhang Z F, Zhao M F, Fu G Y, Ouyang D X. Depinning dynamics of two-dimensional magnetized colloids on a substrate with periodic pinning centers. Physica $A$ 391(10): 2940-2947 (2012)

[14] Ramaswamy S. The mechanics and statistics of active matter. Annu Rev Condens Matter Phys 1(15):323-345 (2010)

[15] Zhao H J, Misko V R, Peeters F M. Dynamics of selforganized driven particles with competing range interaction. Phys Rev E 88(2): 022914 (2013)

[16] Palacci J, Sacanna S, Steinberg A P, Pine D J, Chaikin P M. Living crystals of light-activated colloidal surfers. Science 339(6122): 936-940 (2013)

[17] Zhang T H, Kuipers B W M, Tian W D, Groenewold J, Kegel W K. Polydispersity and gelation in concentrated colloids with competing interactions. Soft Matter 11(2): 297-302 (2015)

[18] Bechinger C, Leonardo R D, Löwen H, Reichhardt C, Volpe G. Active particles in complex and crowded environments. Rev Mod Phys 88(4):045006 (2016)

[19] Reichhardt C, Reichhardt C J O. Depinning and nonequilibrium dynamic phases of particle assemblies driven over random and ordered substrates: a review. Rep Prog Phys 80(2): 026501 (2017)

[20] Cao T T, Li Z, Lv W L, Cao Y G. Living islands of driven two-dimensional magnetic colloids on the disordered substrate. J Phys Commun 1(4): 045008 (2017)

[21] Li F, Josephson D P, Stein A. Colloidal assembly: The road from particles to colloidal molecules and crystals. Angew Chem Int Ed 50(2): 360-388 (2011)

[22] Charbonneau P, Reichman D R. Phase behavior and farfrom-equilibrium gelation in charged attractive colloids. Phys Rev E 75(5): 050401 (2007)

[23] Theurkauff I, Cottin-Bizonne C, Palacci J, Ybert C, Bocquet L. Dynamic clustering in active colloidal suspensions with chemical signaling. Phys Rev Lett 108(26): 268303 (2012)

[24] Mognetti B M, Saric A, Angioletti-Uberti S, Cacciuto A, Valeriani C, Frenkel D. Living clusters and crystals from low-density suspensions of active colloids. Phys Rev Lett 111(24): 245702 (2013) 
[25] Redner G S, Baskaran A, Hagan M F. Reentrant phase behavior in active colloids with attraction. Phys Rev E 88(1): 012305 (2013)

[26] Benassi A, Vanossi A, Santoro G E, Tosatti E. Sliding over a phase transition. Phys Rev Lett 106(25): 256102 (2011)

[27] Steiner P, Roth R, Gnecco E, Baratoff A, Maier S, Glatzel T, Meyer E. Two-dimensional simulation of superlubricity on $\mathrm{NaCl}$ and highly oriented pyrolytic graphite. Phys Rev $B$ 79(4): 045414 (2009)

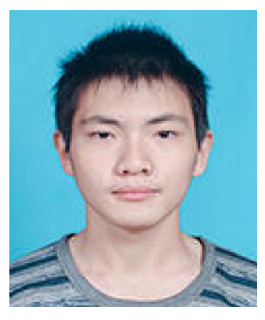

Zhongying ZHANG. He will receive his bachelor degree in physics in 2019 from Zhengzhou University, Zhengzhou, China. He investigated

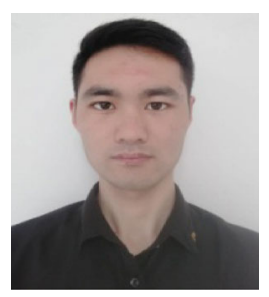

Cange WU. He received his bachelor degree in physics in 2016 from Zhoukou Normal University, Zhoukou, China. He is studying for

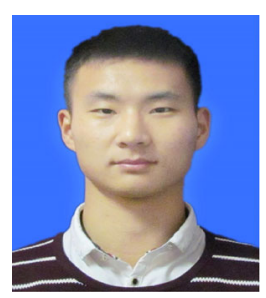

Qi ZHANG. He will receive his bachelor degree in physics in 2019 from Zhengzhou University, Zhengzhou, China. He investigated

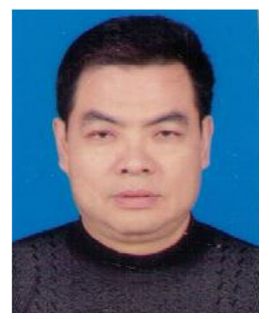

Yigang CAO. He received his Ph.D. degree in condensed matter physics from Zhejiang University, Hangzhou, China, in 2001. His current position
[28] Li X D, Wu C G, Cao T T, Cao Y G. Directional modelocking of driven two-dimensional active magnetized colloids with periodic pinning centers. Physica A 515(2): 279-287 (2019)

[29] Song K N, Wang H L, Ren J, Cao Y G. Interference modelocking of 2D magnetized colloids driven by $\mathrm{dc}$ and ac forces in periodic pinning arrays. Physica A 417(1): 102-109 (2015)

[30] Fisher D S. Flux-lattice melting in thin-film superconductors. Phys Rev B 22(3): 1190-1199 (1980)

the mesoscopic friction under the guidance of professor Yigang Cao in 2017 and 2018. His research interests include mesoscopic friction, gas sensing and sodium batteries.

the M.S. degree at Zhengzhou University. His research interests include colloid and fluid dynamics.

the mesoscopic friction under the guidance of professor Yigang Cao in 2017 and 2018. His research interests include mesoscopic friction and warm dense matter.

is a professor of Zhengzhou University. His research areas cover colloid and fluid dynamics, mesoscopic friction, statistical physics and complex systems. 